\title{
CONDITIONAL RESOLVABILITY IN GRAPHS: A SURVEY
}

\author{
VARAPORN SAENPHOLPHAT and PING ZHANG
}

Received 29 November 2003

\begin{abstract}
For an ordered set $W=\left\{w_{1}, w_{2}, \ldots, w_{k}\right\}$ of vertices and a vertex $v$ in a connected graph $G$, the code of $v$ with respect to $W$ is the $k$-vector $c_{W}(v)=\left(d\left(v, w_{1}\right), d\left(v, w_{2}\right), \ldots, d\left(v, w_{k}\right)\right)$, where $d(x, y)$ represents the distance between the vertices $x$ and $y$. The set $W$ is a resolving set for $G$ if distinct vertices of $G$ have distinct codes with respect to $W$. The minimum cardinality of a resolving set for $G$ is its dimension $\operatorname{dim}(G)$. Many resolving parameters are formed by extending resolving sets to different subjects in graph theory, such as the partition of the vertex set, decomposition, and coloring in graphs, or by combining resolving property with another graph-theoretic property such as being connected, independent, or acyclic. In this paper, we survey results and open questions on the resolving parameters defined by imposing an additional constraint on resolving sets, resolving partitions, or resolving decompositions in graphs.
\end{abstract}

2000 Mathematics Subject Classification: 05C12, 05C20, 05C90.

1. Introduction. We refer to [13] for graphical theoretical notation and terminology not described in this paper. The distance $d(u, v)$ between two vertices $u$ and $v$ in a connected graph $G$ is the length of a shortest $u-v$ path in $G$. For an ordered set $W=\left\{w_{1}, w_{2}, \ldots, w_{k}\right\} \subseteq V(G)$ and a vertex $v$ of $G$, the $k$-vector

$$
c_{W}(v)=\left(d\left(v, w_{1}\right), d\left(v, w_{2}\right), \ldots, d\left(v, w_{k}\right)\right)
$$

is the code of $v$ with respect to $W$. The set $W$ is called a resolving set for $G$ if distinct vertices have distinct codes with respect to $W$. A resolving set containing a minimum number of vertices is a minimum resolving set or a basis for $G$. The (metric) dimension $\operatorname{dim}(G)$ is the number of vertices in a basis for $G$.

For example, consider the graph $G$ shown in Figure 1.1. The ordered set $W_{1}=\left\{v_{1}, v_{3}\right\}$ is not a resolving set for $G$ since $c_{W_{1}}\left(v_{2}\right)=(1,1)=c_{W_{1}}\left(v_{4}\right)$, that is, $G$ contains two vertices with the same code. On the other hand, $W_{2}=\left\{v_{1}, v_{2}, v_{3}\right\}$ is a resolving set for $G$ since the codes for the vertices of $G$ with respect to $W_{2}$ are

$$
\begin{gathered}
c_{W_{2}}\left(v_{1}\right)=(0,1,1), \quad c_{W_{2}}\left(v_{2}\right)=(1,0,1), \quad c_{W_{2}}\left(v_{3}\right)=(1,1,0), \\
c_{W_{2}}\left(v_{4}\right)=(1,2,1), \quad c_{W_{2}}\left(v_{5}\right)=(2,1,1),
\end{gathered}
$$

which are distinct. However, $W_{2}$ is not a minimum resolving set for $G$ since $W_{3}=\left\{v_{1}, v_{2}\right\}$ is also a resolving set. The codes for the vertices of $G$ with respect to $W_{3}$ are

$$
\begin{gathered}
c_{W_{3}}\left(v_{1}\right)=(0,1), \quad c_{W_{3}}\left(v_{2}\right)=(1,0), \quad c_{W_{3}}\left(v_{3}\right)=(1,1), \\
c_{W_{3}}\left(v_{4}\right)=(1,2), \quad c_{W_{3}}\left(v_{5}\right)=(2,1) .
\end{gathered}
$$




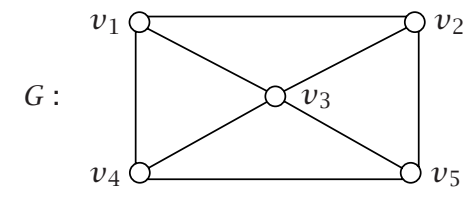

FIGURE 1.1. Illustrating resolving sets.

Since no single vertex constitutes a resolving set for $G$, it follows that $W_{3}$ is a minimum resolving set or a basis for this graph $G$, and $\operatorname{so} \operatorname{dim}(G)=2$.

The example just presented also illustrates an important point. When determining whether a given set $W$ of vertices of a graph $G$ is a resolving set for $G$, we only need to investigate the vertices of $V(G)-W$ since $w \in W$ is the only vertex of $G$ whose distance from $w$ is 0 .

As described in [22], the idea of a resolving set (and of a minimum resolving set) has appeared in the literature previously. In [50], and later in [52], Slater introduced this concept and used locating set for what we have called resolving set. He referred to the cardinality of a minimum resolving set in a graph $G$ as its location number $\operatorname{loc}(G)$. Slater described the usefulness of these ideas when working with U.S. sonar and Coast Guard Loran (Long range aids to navigation) stations. Independently, Harary and Melter [28] discovered the concept of a location number as well, but used the term metric dimension, rather than location number, the terminology that we have adopted. Recently, these concepts were rediscovered by Johnson [32, 33] of the Pharmacia Company while attempting to develop a capability of large datasets of chemical graphs. More applications of this concept to navigation of robots in networks and other areas are discussed in $[4,29,30,31,34]$. It was noted in [27, page 204] that determining the locating number of a graph is an NP-complete problem. Resolving sets in graphs have been studied further in [1, 2, 3, 5, 6, 8, 14, 20, 21, 24, 34, 37, 38]. Recently, these concepts have been extended in various ways and studied for different subjects in graph theory, including such diverse aspects as the partition of the vertex set, decomposition, orientation, domination, coloring in graphs $[15,16,17,19,23,25,35,36,39,47,51]$, and others [22]. Many invariants arising from the study of resolving sets in graph theory offer subjects for applicable research. In this paper, we survey results and open questions on the resolving parameters defined by imposing an additional constraint on resolving sets, resolving partitions, or resolving decompositions in graphs.

2. Conditional resolving sets. Many resolving parameters are formed by combining resolving property with another common graph-theoretic property such as being connected, independent, or acyclic. The generic nature of conditional resolvability in graphs provides various ways of defining new resolving parameters by considering different conditions.

2.1. Connected resolving sets. In general, a connected graph $G$ can have many resolving sets. In this section, we study those resolving sets whose vertices are located "close" to one another. A resolving set $W$ of $G$ is connected if the subgraph $\langle W\rangle$ induced by $W$ is a connected subgraph of $G$. The minimum cardinality of a connected resolving 
set $W$ in a graph $G$ is the connected resolving number $\operatorname{cr}(G)$. A connected resolving set of cardinality $\operatorname{cr}(G)$ is called a cr-set of $G$. This concept was introduced in [43] and further studied in $[42,45,46]$. Since the vertex set $V(G)$ of $G$ is a resolving set and $\langle V(G)\rangle=G$ is connected, $\operatorname{cr}(G)$ is defined for every connected graph $G$. Since every connected resolving set is a resolving set, $\operatorname{dim}(G) \leq \operatorname{cr}(G)$ for all connected graphs $G$. Furthermore, $\operatorname{dim}(G)=\operatorname{cr}(G)$ if and only if $G$ contains a connected basis.

The relationship between cr-sets and bases in a nontrivial connected graph $G$ has been studied in [45]. If $W$ and $W^{\prime}$ are two sets of vertices of a graph $G$ such that $W$ is a resolving set of $G$ and $W \subseteq W^{\prime}$, then $W^{\prime}$ is also a resolving set of $G$. Therefore, if $W$ is a basis of $G$ such that $\langle W\rangle$ is disconnected, then surely there is a smallest superset $W^{\prime}$ of $W$ for which $\left\langle W^{\prime}\right\rangle$ is connected. This suggests the following question: for each basis $W$ of a nontrivial connected graph $G$, does there exist a cr-set $W^{\prime}$ of $G$ such that $W \subseteq W^{\prime}$ ? In [45], it was shown that this question has a negative answer.

Proposition 2.1. There is an infinite class of connected graphs $G$ such that some cr-sets of $G$ contain a basis of $G$ and others contain no basis of $G$.

Proposition 2.1 suggests yet another question. For each connected graph $G$, do there exist some cr-set $W^{\prime}$ and some basis $W$ of $G$ with $W \subseteq W^{\prime}$ ? It was shown in [45] that even this question has a negative answer as well, by presenting a stronger result. Let $X$ and $Y$ be two sets of vertices in a connected graph $G$. The distance between $X$ and $Y$ is defined as

$$
d(X, Y)=\min \{d(x, y) \mid x \in X \text { and } y \in Y\} .
$$

THEOREM 2.2. For each positive integer $N$, there is an infinite class of connected graphs $G$ such that $d(W, S) \geq N$ for every basis $W$ of $G$ and every cr-set $S$ of $G$.

The following three results give relationships between cr-sets and bases in some wellknown classes of graphs, namely, complete graphs, complete bipartite graphs, cycles, and trees.

Proposition 2.3. If $G$ is a complete graph of order at least 3 or a complete bipartite graph that is not a star, then a set $W$ of vertices of $G$ is a basis of $G$ if and only if $W$ is a cr-set of $G$.

Proposition 2.4. For a cycle $C_{n}$ of order $n \geq 4$, every $\mathrm{cr}$-set of $C_{n}$ is a basis of $C_{n}$.

The converse of Proposition 2.4 is not true for $n \geq 5$ since some basis of $C_{n}$ consists of two nonadjacent vertices of $C_{n}$ and, therefore, it is not a cr-set of $C_{n}$.

Proposition 2.5. If $T$ is a tree that is not a path, then every $\mathrm{cr}-$ set of $T$ contains a basis of $T$ as a proper subset.

If $G$ is a connected graph of order $n$, then every set of $n-1$ vertices of $G$ is a resolving set of $G$. Moreover, every nontrivial connected graph $G$ contains a vertex $v$ that is not a cut-vertex, and so $V(G)-\{v\}$ is a connected resolving set for $G$. Thus

$$
1 \leq \operatorname{cr}(G) \leq n-1
$$


for all connected graphs $G$ of order $n \geq 3$. The lower and upper bounds in (2.2) are both sharp. In fact, all connected graphs $G$ of order $n \geq 2$ with $\operatorname{cr}(G)=1, n-1$ are characterized in [43].

THEOREM 2.6. Let $G$ be a connected graph of order $n \geq 2$. Then

(a) $\operatorname{cr}(G)=1$ if and only if $G=P_{n}$,

(b) $\operatorname{cr}(G)=n-1$ if and only if $G=K_{n}$ or $G=K_{1, n-1}$.

Moreover, for each pair $k, n$ with $1 \leq k \leq n-1$, there is a connected graph of order $n$ with connected resolving number $k$.

If $G$ is a connected graph with $\operatorname{dim}(G)=a$ and $\operatorname{cr}(G)=b$, then $1 \leq a \leq b$. On the other hand, all pairs $a, b$ of positive integers with $a \leq b$ that can be realizable as the dimension and connected resolving number of some connected graph have been determined in [43].

THEOREM 2.7. For positive integers $a, b$ with $a \leq b$, there exists a connected graph $G$ with $\operatorname{dim}(G)=a$ and $\operatorname{cr}(G)=b$ if and only if $(a, b) \notin\{(1, k): k \geq 2\}$.

All triples $a, b, n$ of positive integers that can be realized as the dimension, connected resolving number, and order, respectively, of some connected graph have also been determined in [43].

THEOREM 2.8. Let $a, b, n$ be integers with $n \geq 5$. Then there exists a connected graph $G$ of order $n$ such that $\operatorname{dim}(G)=a$ and $\operatorname{cr}(G)=b$ if and only if $a, b, n$ satisfy one of the following:

(a) $a=b=1$,

(b) $b=n-1$ and $a \in\{n-2, n-1\}$,

(c) $2 \leq a \leq b \leq n-2$.

In general, the graphs we have seen have had several cr-sets. It was shown in [46] that for every integer $k \geq 2$, there exists a graph with a unique cr-set of cardinality $k$. Furthermore, this result can be extended to the following.

THEOREM 2.9. For every pair $r$, $k$ of integers with $k \geq 2$ and $0 \leq r \leq k$, there exists $a$ connected graph $G$ such that $\operatorname{cr}(G)=k$ and exactly $r$ vertices of $G$ belong to every $\mathrm{cr}-$ set of $G$.

A resolving set $W$ of a nontrivial connected $G$ is a minimal resolving set if no proper subset of $W$ is a resolving set. The maximum cardinality of a minimal resolving set is the upper dimension $\operatorname{dim}^{+}(G)$. A minimal resolving set of cardinality $\operatorname{dim}^{+}(G)$ is an upper basis for $G$. Since every minimum resolving set is a minimal resolving set for $G$, it follows that $\operatorname{dim}(G) \leq \operatorname{dim}^{+}(G)$. These concepts were introduced and studied in [14]. Similarly, a connected resolving set $W$ of $G$ is a minimal connected resolving set if no proper subset of $W$ is a connected resolving set. The maximum cardinality of a minimal connected resolving set is the upper connected resolving number $\mathrm{cr}^{+}(G)$. These concepts were introduced in [43] and further studied in [42, 46]. Certainly, if $G$ is a nontrivial connected graph of order $n$, then

$$
1 \leq \operatorname{cr}(G) \leq \operatorname{cr}^{+}(G) \leq n-1 .
$$


It was shown in [46] that there is no "intermediate value theorem" for minimal connected resolving sets, that is, if $k$ is an integer such that $\operatorname{cr}(G)<k<\mathrm{cr}^{+}(G)$, then there need not exist a minimal connected resolving set of cardinality $k$ in $G$.

In order to present the connected resolving numbers and upper connected resolving numbers of some well-known classes of graphs, we need some additional definitions and notation. A vertex of degree at least 3 in a graph $G$ will be called a major vertex. An endvertex $u$ of $G$ is said to be a terminal vertex of a major vertex $v$ of $G$ if $d(u, v)<$ $d(u, w)$ for every other major vertex $w$ of $G$. The terminal degree $\operatorname{ter}(v)$ of a major vertex $v$ is the number of terminal vertices of $v$. A major vertex $v$ of $G$ is an exterior major vertex of $G$ if it has positive terminal degree. Let $\sigma(G)$ denote the sum of the terminal degrees of the major vertices of $G$ and let ex $(G)$ denote the number of exterior major vertices of $G$. In fact, $\sigma(G)$ is the number of endvertices of $G$.

(a) If $G=K_{n}$ for $n \geq 3$ or $G=K_{1, n-1}$ for $n \geq 4$, then $\operatorname{cr}^{+}(G)=\operatorname{cr}(G)=n-1$.

(b) If $G=P_{n}$ for $n \geq 2$, then $\operatorname{cr}^{+}(G)=\operatorname{cr}(G)=1$.

(c) If $G=C_{n}$ for $n \geq 4$, then $\operatorname{cr}^{+}(G)=\operatorname{cr}(G)=2$.

(d) For $k \geq 2$, let $G=K_{n_{1}, n_{2}, \ldots, n_{k}}$ be a complete $k$-partite graph that is not a star. Let $n=n_{1}+n_{2}+\cdots+n_{k}$ and let $\ell$ be the number of 1 's in $\left\{n_{i}: 1 \leq i \leq k\right\}$. Then

$$
\operatorname{cr}^{+}(G)=\operatorname{cr}(G)= \begin{cases}n-k & \text { if } \ell=0, \\ n-k+\ell-1 & \text { if } \ell \geq 1 .\end{cases}
$$

(e) Let $T$ be a tree that is not a path, having order $n \geq 4$ and $p$ exterior major vertices $v_{1}, v_{2}, \ldots, v_{p}$. For $1 \leq i \leq p$, let $u_{i 1}, u_{i 2}, \ldots, u_{i k_{i}}$ be the terminal vertices of $v_{i}$ and let $\ell_{i j}=d\left(v_{i}, u_{i j}\right)\left(1 \leq j \leq k_{i}\right)$. Then

$$
\operatorname{cr}^{+}(T)=\operatorname{cr}(T)=n+\sigma(T)-\operatorname{ex}(T)-\sum_{i, j} \ell_{i j}
$$

We have seen in Theorem 2.6 that $K_{n}$ and $K_{1, n-1}$ are the only connected graphs of order $n \geq 4$ with connected resolving number $n-1$. In fact, this is also true for the upper connected numbers of graphs [46].

THEOREM 2.10. Let $G$ be a connected graph of order $n \geq 4$. Then $\operatorname{cr}^{+}(G)=n-1$ if and only if $G=K_{n}$ or $G=K_{1, n-1}$.

Note that every graph $G$ encountered thus far has the property that either $\operatorname{cr}^{+}(G)=$ $\operatorname{cr}(G)$ or $\operatorname{cr}^{+}(G)-\operatorname{cr}(G) \leq 2$. This might lead one to believe that $\operatorname{cr}^{+}(G)$ and $\operatorname{cr}(G)$ are close for every connected graph $G$. However, this is not the case. In fact, every pair $a$, $b$ of integers with $2 \leq a \leq b$ is realizable as the connected resolving number and upper connected resolving number of some graph, as shown in [42].

THEOREM 2.11. Let $a$ and $b$ be integers with $1 \leq a \leq b$. Then there exists a connected graph $G$ with $\operatorname{cr}(G)=a$ and $\operatorname{cr}^{+}(G)=b$ if and only if $(a, b) \neq(1, i)$ for all $i \geq 2$.

2.2. Independent resolving sets. Independent sets of vertices in graphs form one of the most commonly studied concepts in graph theory. The independent sets of maximum cardinality are called maximum independent sets and these are the independent 
sets that have received the most attention. The number of vertices in a maximum independent set in a graph $G$ is the independence number (or vertex independence number) of $G$ and is denoted by $\beta(G)$. Some graphs $G$ contain (ordered) independent sets $W$ such that the vertices of $G$ are uniquely distinguished by their distances from the vertices of $W$. In [18], we study the existence of such independent sets in graphs and, when they exist, investigate the minimum cardinality of such a set.

An independent resolving set $W$ in a connected graph $G$ is both resolving and independent. The cardinality of a minimum independent resolving set (or simply an ir-set) in a graph $G$ is the independent resolving number $\operatorname{ir}(G)$. This concept was introduced and studied in [18]. Let $G$ be a connected graph of order $n$ containing an independent resolving set. Since every independent resolving set of $G$ is a resolving set, it follows that

$$
1 \leq \operatorname{dim}(G) \leq \operatorname{ir}(G) \leq \beta(G) \leq n-1
$$

A lower bound for $\operatorname{ir}(G)$ of a connected graph $G$ in terms of its maximum degree $\Delta(G)$ was given in [18].

Proposition 2.12. If $G$ is a nontrivial connected graph for which $\operatorname{ir}(G)$ is defined, then

$$
\operatorname{ir}(G) \geq\left\lceil\log _{3}(\Delta(G)+1)\right\rceil .
$$

Furthermore, this bound is sharp.

Not all graphs have an independent resolving set, however, and so $\operatorname{ir}(G)$ is not defined for all graphs $G$. In order to present the results on the existence of independent resolving sets in graphs, we need some additional definitions. Two vertices $u$ and $v$ in a connected graph $G$ are distance-similar if $d(u, x)=d(v, x)$ for all $x \in V(G)-\{u, v\}$. For a vertex $v$ in a graph $G$, let $N(v)$ be the set of vertices adjacent to $v$ and let $N[v]=N(v) \cup\{v\}$. Then two vertices $u$ and $v$ in a connected graph are distance-similar if and only if (1) $u v \notin E(G)$ and $N(u)=N(v)$ or (2) $u v \in E(G)$ and $N[u]=N[v]$. Distance similarity in a graph $G$ is an equivalence relation on $V(G)$. The following observations are useful.

OBSERVATION 2.13. If $U$ is a distance-similar equivalence class in a connected graph $G$ with $|U|=p \geq 2$, then every resolving set of $G$ contains at least $p-1$ vertices from $U$. Thus, if $G$ has $k$ distance-similar equivalence classes and $\operatorname{ir}(G)$ is defined, then

$$
n-k \leq \operatorname{dim}(G) \leq \operatorname{ir}(G) .
$$

ObSERVATION 2.14. Let $G$ be a connected graph and let $U$ be a distance-similar equivalence class in $G$ with $|U| \geq 3$. If $U$ is not independent in $G$, then $\operatorname{ir}(G)$ is not defined.

The converse of Observation 2.14 is not true. For example, let $G=K_{3,3}$ with partite sets $V_{1}$ and $V_{2}$. Then $\operatorname{ir}(G)$ is not defined. On the other hand, $V_{1}$ and $V_{2}$ are the only 
distance-similar equivalence classes and they are both independent. The following result appears in [18].

Proposition 2.15. Let $G$ be a connected graph of order $n \geq 6$ for which $\operatorname{ir}(G)$ is defined. If $W$ is an independent resolving set of $G$, then $\operatorname{deg} w \leq n-3$ for every $w \in W$.

The following corollary is a consequence of Proposition 2.15.

COROLLARY 2.16. Let $G$ be a connected graph of order $n \geq 6$.

(a) If $G$ contains two nonadjacent vertices of degree $n-2$, then $\operatorname{ir}(G)$ is not defined.

(b) If $G$ contains two vertices of degree $n-1$, then $\operatorname{ir}(G)$ is not defined.

On the other hand, there exist graphs $G$ of order $n \geq 6$ having two adjacent vertices of degree $n-2$ for which $\operatorname{ir}(G)$ is defined. For example, let $G$ be the graph obtained from $\bar{K}_{n-2}$, where $V\left(\bar{K}_{n-2}\right)=\left\{v_{1}, v_{2}, \ldots, v_{n-2}\right\}$, and $P_{2}: x, y$ by adding the edges $x v_{i}$ $(1 \leq i \leq n-3)$ and $y v_{j}(2 \leq j \leq n-2)$. Then $W=\left\{v_{1}, v_{2}, \ldots, v_{n-4}\right\}$ is a minimum independent resolving set of $G$, and so $\operatorname{ir}(G)=n-4$.

Proposition 2.17. Let $G$ be a connected graph of order $n \geq 4$. Suppose that $G$ contains two distinct distance-similar equivalence classes $U_{1}$ and $U_{2}$ of cardinality at least 2. If some vertex of $U_{1}$ is adjacent to a vertex of $U_{2}$, then $\operatorname{ir}(G)$ is not defined.

The converse of Proposition 2.17 is not true. For example, let $G$ be the graph obtained from two copies of $K_{4}$, whose vertex sets are $U_{1}=\left\{u_{1}, u_{2}, u_{3}, u_{4}\right\}$ and $V_{1}=$ $\left\{v_{1}, v_{2}, v_{3}, v_{4}\right\}$, by adding the edge $u_{4} v_{4}$. Then $U_{1}-\left\{u_{4}\right\}$ and $V_{1}-\left\{v_{4}\right\}$ are two distinct distance-similar equivalence classes of $G$. By Observation 2.14, $\operatorname{ir}(G)$ does not exist. However, no edge joins a vertex in $U_{1}-\left\{u_{4}\right\}$ and a vertex in $V_{1}-\left\{v_{4}\right\}$.

The existence of independent resolving sets in some well-known classes of graphs is determined in [18]. If $G$ is a nontrivial connected graph of order $n$ for which $\operatorname{ir}(G)$ exists, then $1 \leq \operatorname{ir}(G) \leq n-1$. The following result characterizes all nontrivial connected graphs $G$ of order $n$ for which $\operatorname{ir}(G) \in\{1, n-2, n-1\}$.

THEOREM 2.18. Let $G$ be a connected graph of order $n \geq 2$ for which $\operatorname{ir}(G)$ exists. Then

(a) $\operatorname{ir}(G)=1$ if and only if $G=P_{n}$,

(b) $\operatorname{ir}(G)=n-2$ if and only if $n \geq 3$ and $G=K_{1, n-1}$ or $n=4$ and $G=\left(K_{2} \cup K_{1}\right)+K_{1}$,

(c) $\operatorname{ir}(G)=n-1$ if and only if $n=2$ and $G=K_{2}$.

All pairs $k, n$ of positive integers with $k \leq n$ that are realizable as the independent resolving number and the order of some connected graph are also determined in [18].

THEOREM 2.19. For each pair $k, n$ of positive integers with $k \leq n$, there exists a connected graph $G$ of order $n$ with $\operatorname{ir}(G)=k$ if and only if $(k, n)=(1,2)$ or $1 \leq k \leq n-2$.

It was also shown in [18] that certain pairs $a, b$ are realizable as the dimension and the independent resolving number of some connected graph.

THEOREM 2.20. For every pair $a, b$ of integers with $2 \leq a \leq b \leq\lfloor 3 a / 2\rfloor$, there exists a connected graph $G$ such that $\operatorname{dim}(G)=a$ and $\operatorname{ir}(G)=b$. 
By Theorem 2.20, every pair $a, b$ of positive integers with $2 \leq a \leq b \leq\lfloor 3 a / 2\rfloor$ is realizable as the dimension and the independent resolving number of some connected graph. Furthermore, for each pair $a, b$ of positive integers with $4 \leq a \leq b$, it can be shown that (1) there exists a connected graph $F$ with $\operatorname{dim}(F)=\operatorname{ir}(F)=a$ and $\beta(F)=b$, (2) there exists a connected graph $G$ with $\operatorname{dim}(G)=a$ and $\beta(G)=b$ such that $\operatorname{dim}(G) \neq$ $\operatorname{ir}(G)$, (3) there exists a connected graph $H$ with $\operatorname{ir}(H)=a$ and $\beta(H)=b$ such that $\operatorname{dim}(H) \neq \operatorname{ir}(H)$. However, we do not have a complete solution to the following problem.

Problem 2.21. For which triples $a, b, c$ of positive integers with $2 \leq a \leq b \leq c$ does there exist a connected graph $G$ such that $\operatorname{dim}(G)=a, \operatorname{ir}(G)=b$, and $\beta(G)=c$ ?

3. Conditional resolving partitions. As described in [9, 10], dividing the vertex set of a graph into classes according to some prescribed rule is a fundamental process in graph theory. For example, the vertices of a graph can be divided into cut-vertices and non-cut-vertices. Equivalently, the vertices of a tree are divided into its leaves and nonleaves. The vertices of a graph can also be partitioned according to the degrees of its vertices. When studying distance, the vertices of a connected graph are partitioned according to their distance from the root. Perhaps the best known example of this process, however, is graph coloring, where the vertex set of a graph is partitioned into classes each of which is independent in the graph. In [19], the vertices of a connected graph $G$ are represented by other means, namely, through partitions of $V(G)$ and the distances between each vertex of $G$ and the subsets in the partition.

For a set $S$ of vertices of a connected graph $G$ and a vertex $v$ of $G$, the distance $d(v, S)$ between $v$ and $S$ is defined as

$$
d(v, S)=\min \{d(v, x): x \in S\}
$$

For an ordered $k$-partition $\Pi=\left\{S_{1}, S_{2}, \ldots, S_{k}\right\}$ of $V(G)$ and a vertex $v$ of $G$, the code of $v$ with respect to $\Pi$ is defined as the $k$-vector

$$
c_{\Pi}(v)=\left(d\left(v, S_{1}\right), d\left(v, S_{2}\right), \ldots, d\left(v, S_{k}\right)\right) .
$$

The partition $\Pi$ is called a resolving partition for $G$ if the distinct vertices of $G$ have distinct codes with respect to $\Pi$. The minimum $k$ for which there is a resolving $k$ partition of $V(G)$ is the partition dimension $\operatorname{pd}(G)$ of $G$.

As an illustration of these concepts, consider the graph $G$ in Figure 3.1.

Let $\Pi_{1}=\left\{S_{1}, S_{2}, S_{3}, S_{4}\right\}$, where $S_{1}=\left\{v_{1}, v_{2}\right\}, S_{2}=\left\{v_{3}\right\}, S_{3}=\left\{v_{4}\right\}$, and $S_{4}=\left\{v_{5}\right\}$. Then the five codes with respect to $\Pi_{1}$ are

$$
\begin{gathered}
c_{\Pi_{1}}\left(v_{1}\right)=(0,1,1,1), \quad c_{\Pi_{1}}\left(v_{2}\right)=(0,1,2,2), \quad c_{\Pi_{1}}\left(v_{3}\right)=(1,0,1,2), \\
c_{\Pi_{1}}\left(v_{4}\right)=(1,1,0,1), \quad c_{\Pi_{1}}\left(v_{5}\right)=(1,2,1,0) .
\end{gathered}
$$




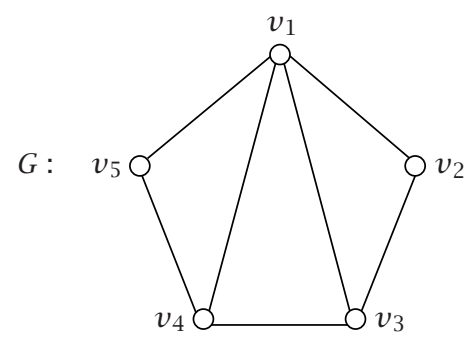

FIGURE 3.1. Illustrating resolving partitions.

Since the five codes are distinct, $\Pi_{1}$ is a resolving partition of $G$. However, $\Pi_{1}$ is not a minimum resolving partition of $G$. To see this, let $\Pi_{2}=\left\{S_{1}, S_{2}, S_{3}\right\}$, where $S_{1}=\left\{v_{1}, v_{2}\right\}$, $S_{2}=\left\{v_{3}\right\}$, and $S_{3}=\left\{v_{4}, v_{5}\right\}$. Then the corresponding codes are

$$
\begin{gathered}
c_{\Pi_{2}}\left(v_{1}\right)=(0,1,1), \quad c_{\Pi_{2}}\left(v_{2}\right)=(0,1,2), \quad c_{\Pi_{2}}\left(v_{3}\right)=(1,0,1), \\
c_{\Pi_{2}}\left(v_{4}\right)=(1,1,0), \quad c_{\Pi_{2}}\left(v_{5}\right)=(1,2,0) .
\end{gathered}
$$

So, $\Pi_{2}$ is a resolving partition of $G$. Moreover, since no 2-partition is a resolving partition of $G$, it follows that $\Pi_{2}$ is a minimum resolving partition of $G$, and $\operatorname{so} \operatorname{pd}(G)=3$. The resolving partition and partition dimension of a graph were introduced and studied in $[19,23]$.

3.1. Connected resolving partitions. For a connected graph $G$, a resolving partition $\Pi=\left\{S_{1}, S_{2}, \ldots, S_{k}\right\}$ of $V(G)$ is defined to be connected in [41] if the subgraph $\left\langle S_{i}\right\rangle$ induced by each subset $S_{i}(1 \leq i \leq k)$ is connected in $G$. The minimum $k$ for which there is a connected resolving $k$-partition of $V(G)$ is the connected partition dimension $\operatorname{cpd}(G)$ of $G$. A connected resolving partition of $V(G)$ containing $\operatorname{cpd}(G)$ elements is called a minimum connected resolving partition (or cr-partition) of $V(G)$. If $G$ is a nontrivial connected graph with $V(G)=\left\{v_{1}, v_{2}, \ldots, v_{n}\right\}$, then the $n$-partition $\left\{S_{1}, S_{2}, \ldots, S_{n}\right\}$, where $S_{i}=\left\{v_{i}\right\}$ for $1 \leq i \leq n$, is a connected resolving partition for $G$. Thus, $\operatorname{cpd}(G)$ is defined for every nontrivial connected graph $G$. Certainly, every connected resolving partition of a connected graph is a resolving partition. Thus, if $G$ is a connected graph of order $n \geq 2$, then

$$
2 \leq \operatorname{pd}(G) \leq \operatorname{cpd}(G) \leq n
$$

Moreover, $\operatorname{pd}(G)=\operatorname{cpd}(G)$ if and only if $G$ contains a minimum resolving partition that is connected.

Let $\Pi$ be a partition of $V(G)$. A partition $\Pi^{\prime}$ of $V(G)$ is called a refinement of $\Pi$ if each element of $\Pi^{\prime}$ is a subset of some element of $\Pi$. It was shown in [41] that every refinement of a resolving partition of a connected graph $G$ is also a resolving partition of $G$. If we are given a minimum resolving partition $\Pi$ of a connected graph $G$, then we can find a connected resolving partition $\Pi^{\prime}$ of $G$, where $\Pi^{\prime}$ is a refinement of $\Pi$. Indeed, the partition each of whose elements consists of a single vertex has this property. However, $\Pi^{\prime}$ need not be a minimum connected resolving partition for $G$. In fact, it 
may be the case that no minimum connected resolving partition is a refinement of any minimum resolving partition of $V(G)$.

Upper and lower bounds for the connected partition dimension of a connected graph in terms of its order and diameter were established in [41]. For integers $n$ and $d$ with $1 \leq d<n$, we define $g(n, d)$ as the least positive integer $k$ for which $k d^{k-1} \geq n$. Thus $g(n, 1)=n$ for all $n \geq 2$.

THEOREM 3.1. If $G$ is a connected graph of order $n \geq 3$ and diameter $d$, then

$$
g(n, d) \leq \operatorname{pd}(G) \leq \operatorname{cpd}(G) \leq n-d+1 .
$$

It was shown in [19] that for each integer $n \geq 2$, the path $P_{n}$ of order $n$ is the only connected graph of order $n$ having partition dimension 2, the complete graph $K_{n}$ is the only connected graph of order $n$ having partition dimension $n$, and the graphs $K_{1, n-1}$, $K_{n}-e, K_{1}+\left(K_{1} \cup K_{n-2}\right)$ are the only connected graphs of order $n \geq 3$ with partition dimension $n-1$. Those graphs are also the only connected graphs of order $n \geq 3$ with connected partition dimension $2, n$, or $n-1$, respectively (see [41]).

THEOREM 3.2. Let $G$ be a connected graph of order $n \geq 3$. Then

(a) $\operatorname{cpd}(G)=2$ if and only if $G=P_{n}$,

(b) $\operatorname{cpd}(G)=n$ if and only if $G=K_{n}$,

(c) $\operatorname{cpd}(G)=n-1$ if and only if $G \in\left\{K_{1, n-1}, K_{n}-e, K_{1}+\left(K_{1} \cup K_{n-2}\right)\right\}$.

We have seen that if $G$ is a nontrivial connected graph with $\operatorname{pd}(G)=a$ and $\operatorname{cpd}(G)=b$, then $2 \leq a \leq b$. Furthermore, the nontrivial paths are the only nontrivial connected graphs with partition dimension 2 and connected partition dimension 2 . Thus, $\operatorname{pd}(G)=$ 2 if and only if $\operatorname{cpd}(G)=2$ if and only if $G=P_{n}$. On the other hand, it was shown in [41] that every pair $a, b$ of integers with $3 \leq a \leq b$ is realizable as the partition dimension and the connected partition dimension, respectively, of some connected graph, as stated next.

THEOREM 3.3. For every pair $a, b$ of integers with $3 \leq a \leq b$, there is a connected graph $G$ with $\operatorname{pd}(G)=a$ and $\operatorname{cpd}(G)=b$.

The partition dimensions of some special types of trees that are not paths were studied in [23]. There is no general formula, however, for the partition dimension of a tree that is not a path. On the other hand, a formula for the connected partition dimension of a tree that is not a path was established in [41]. Recall that $\sigma(G)$ denotes the sum of the terminal degrees of the major vertices of a graph $G$ and $\operatorname{ex}(G) \operatorname{denotes}$ the number of exterior major vertices of $G$, as defined in Section 2.1.

THEOREM 3.4. If $T$ is a tree that is not a path, then

$$
\operatorname{cpd}(T)=\sigma(T)-\operatorname{ex}(T)+1 .
$$

3.2. Independent resolving partitions. For a connected graph $G$, a $k$-partition $\Pi=$ $\left\{S_{1}, S_{2}, \ldots, S_{k}\right\}$ is independent if the subgraph $\left\langle S_{i}\right\rangle$ induced by $S_{i}$ is independent in $G$ for each $i(1 \leq i \leq k)$. Partitions of $V(G)$ that are both resolving and independent were studied in [5,6] and called resolving-colorings (or locating-colorings) since the 
coloring of $G$ obtained by coloring each vertex of $S_{i}(1 \leq i \leq k)$ the color $i$ is a proper coloring. The resolving-chromatic number (or locating-chromatic number) $\chi_{r}(G)$ of $G$ is the minimum $k$ for which there is a resolving, independent $k$-partition of $V(G)$. It was observed in [5] that for every connected graph $G$,

$$
\chi(G) \leq \chi_{r}(G) \leq \chi(G)+\operatorname{dim}(G)
$$

where $\chi(G)$ is the chromatic number of $G$. The following result appears in [5].

THEOREM 3.5. For every connected graph $G$ of order $n \geq 3$,

$$
3 \leq \chi_{r}(G) \leq n
$$

Moreover, $\chi_{r}(G)=n$ if and only if $G$ is a complete multipartite graph.

It was shown in [6] that if $G$ is a connected graph of order $n \geq 3$ containing an induced complete multipartite subgraph of order $n-1$, then $(n+1) / 2 \leq \chi_{r}(G) \leq n$ and, furthermore, for each integer $k$ with $(n+1) / 2 \leq k \leq n$, there exists such a graph $G$ of order $n$ with $\chi_{r}(G)=k$. Graphs of order $n$ containing an induced complete multipartite subgraph of order $n-1$ are used to characterize all connected graphs of order $n \geq 4$ with resolving-chromatic number $n-1$.

Bounds for the resolving-chromatic number of a graph were established in [5] in terms of its order and diameter.

THEOREM 3.6. If $G$ is a connected graph of order $n \geq 3$ and diameter $d \geq 2$, then

$$
\log _{d+1} n \leq \chi_{r}(G) \leq n-d+2
$$

THEOREM 3.7. If $G$ is a connected graph of order $n \geq 3$, diameter $d \geq 2$, and $\chi_{r}(G)=k$, then $n \leq k d^{k-1}-1$.

We have seen that if $G$ is a nontrivial connected graph with $\chi(G)=a$ and $\chi_{r}(G)=b$, then $2 \leq a \leq b$. It was shown in [5] that every pair $a, b$ of integers with $2 \leq a \leq b$ is realizable as the chromatic number and resolving-chromatic number, respectively, of some connected graph.

THEOREM 3.8. For each pair $a, b$ of integers with $2 \leq a \leq b$, there exists a connected graph $G$ with $\chi(G)=a$ and $\chi_{r}(G)=b$.

In [5], the resolving-chromatic numbers of some well-known classes of graphs are determined. Furthermore, the resolving-chromatic number of a tree has been studied.

THEOREM 3.9. Let $n \geq 5$. There exists a tree of order $n$ having resolving-chromatic number $k$ if and only if $k \in\{3,4, \ldots, n-2, n\}$.

THEOREM 3.10. Let $k \geq 3$. If $T$ is a tree for which $\Delta(T)>(k-1) 2^{k-2}$, then $\chi_{r}(T)>k$.

THEOREM 3.11. Let $\mathcal{T}$ be the class of all trees for which $\Delta(T)=4$ and $\chi_{r}(T)=3$. Then $\mathcal{T}$ is infinite. Furthermore, if $T \in \mathcal{T}$, then $T$ contains a unique vertex of degree 4. 
3.3. Acyclic resolving partitions. A graph $G$ is acyclic if $G$ has no cycles. Every independent set $S$ of vertices in a graph $G$ has the property that $\langle S\rangle$ is acyclic, and many results concerning the chromatic number of $G$ have been extended to partitions of $V(G)$ in which each subset induces an acyclic subgraph. It is natural, therefore, to study resolving partitions in which each subset induces an acyclic subgraph. For a connected graph $G$, a $k$-partition $\Pi=\left\{S_{1}, S_{2}, \ldots, S_{k}\right\}$ of $V(G)$ is acyclic if the subgraph $\left\langle S_{i}\right\rangle$ induced by $S_{i}$ is acyclic in $G$ for each $i(1 \leq i \leq k)$. The vertex-arboricity $a(G)$ of $G$ is defined in $[11,12]$ as the minimum $k$ such that $V(G)$ has an acyclic $k$-partition. If an acyclic partition $\Pi$ of $V(G)$ is also a resolving partition, then $\Pi$ is called a resolving acyclic partition of $G$, which was introduced and studied in $[48,49]$. The minimum $k$ for which $G$ contains a resolving acyclic $k$-partition is the acyclic partition dimension $\operatorname{apd}(G)$ of $G$. Since every resolving acyclic partition is an acyclic partition,

$$
a(G) \leq \operatorname{apd}(G)
$$

for each connected graph $G$. Furthermore, for every connected graph $G$ of order $n \geq 2$,

$$
2 \leq \operatorname{pd}(G) \leq \operatorname{apd}(G) \leq \chi_{r}(G) \leq n .
$$

In particular, if $G$ is a tree, then $\operatorname{pd}(G)=\operatorname{apd}(G)$. All connected graphs of order $n \geq 2$ with acyclic partition dimension 2, $n-1$, or $n$ are determined in [49].

THEOREM 3.12. Let $G$ be a connected graph of order $n \geq 2$. Then

(a) $\operatorname{apd}(G)=2$ if and only if $G=P_{n}$,

(b) $\operatorname{apd}(G)=n$ if and only if $G=K_{n}$,

(c) for $n \geq 5, \operatorname{apd}(G)=n-1$ if and only if

$$
G \in\left\{C_{4}+K_{1}, K_{1, n-1}, K_{n}-e, K_{1}+\left(K_{1} \cup K_{n-2}\right)\right\} .
$$

Relationships between the acyclic partition dimensions of a connected graph and other graphical parameters, including arboricity, partition dimension, dimension, and resolving-chromatic number, are studied in [48]. The next two results present bounds for the acyclic partition dimension of a connected graph in terms of (1) its resolvingchromatic number, (2) its partition dimension and arboricity, (3) its dimension and arboricity (see [48]).

THEOREM 3.13. For every nontrivial connected graph $G$,

$$
\frac{\chi_{r}(G)}{2} \leq \operatorname{apd}(G) \leq \chi_{r}(G) .
$$

Moreover, for every pair $a, b$ of integers with $a \geq 2$ and $b / 2<a \leq b$, there exists $a$ connected graph $G$ with $\operatorname{apd}(G)=a$ and $\chi_{r}(G)=b$.

However, it is not known whether there exists a connected graph $G$ such that $\chi_{r}(G)=$ $2 \operatorname{apd}(G)$.

THEOREM 3.14. For every nontrivial connected graph $G$,

$$
\operatorname{apd}(G) \leq a(G) \operatorname{pd}(G), \quad \operatorname{apd}(G) \leq a(G)+\operatorname{dim}(G) .
$$


However, just how good the upper bounds in Theorem 3.14 are is not known. The girth of a graph (with cycles) is the length of its shortest cycle. Bounds for the acyclic partition dimension of a connected graph in terms of its order and girth were established in [48].

THEOREM 3.15. If $G$ is a connected graph of order $n \geq 3$ and girth $\ell \geq 3$, then

$$
3 \leq \operatorname{apd}(G) \leq n-\ell+3
$$

Furthermore, if $G$ is a cycle of order $n \geq 3$, then $\operatorname{apd}(G)=3$. On the other hand, $\operatorname{apd}(G)=$ $n-\ell+3$ if and only if $G=K_{n}$ or $G=C_{n}$.

We have seen that if $G$ is a connected graph of order $n \geq 2$, then $2 \leq \operatorname{pd}(G) \leq$ $\operatorname{apd}(G) \leq \chi_{r}(G) \leq n$. It was shown in $[5,19]$ that $\operatorname{pd}\left(K_{n}\right)=\chi_{r}\left(K_{n}\right)=n$ and $\operatorname{pd}\left(P_{n}\right)=$ $\chi_{r}\left(P_{n}\right)=2$. Moreover, for $n \geq 3, \operatorname{pd}\left(C_{n}\right)=3$, while $\chi_{r}\left(C_{n}\right)=3$ if $n$ is odd and $\chi_{r}\left(C_{n}\right)=4$ if $n$ is even. From Theorems 3.12 and 3.15, we then have the following (see [48]).

COROLlaRY 3.16. If $G=K_{n}, P_{n}$ for $n \geq 2$ or $G=C_{n}$ for each odd integer $n \geq 3$, then

$$
\operatorname{pd}(G)=\operatorname{apd}(G)=\chi_{r}(G) .
$$

It is not known whether Corollary 3.16 is also true for other graphs.

The clique number of a graph is the maximum order among the complete subgraphs of the graph. A lower bound for the acyclic partition dimension of a connected graph in terms of its clique number is given in [48].

THEOREM 3.17. For a connected graph $G$ with the clique number $\omega$,

$$
\operatorname{apd}(G) \geq\left\lceil\frac{\omega}{2}\right\rceil+1
$$

Moreover, for each integer $\omega \geq 2$, there exists a connected graph $G_{\omega}$ having clique number $\omega$ such that $\operatorname{apd}\left(G_{\omega}\right)=\lceil\omega / 2\rceil+1$.

If $G$ is a nontrivial connected graph of order $n$ and diameter $d$, then

$$
2 \leq \operatorname{apd}(G) \leq n-d+1
$$

Furthermore, it was shown in [48] that for each triple $d, k, n$ of integers with $2 \leq d \leq$ $n-2$ and $3 \leq(n-d+1) / 2 \leq k \leq n-d+1$, there exists a connected graph of order $n$ having diameter $d$ and acyclic partition dimension $k$. On the other hand, for those triples $d, k, n$ of integers with $2 \leq d \leq n-2$ and $3 \leq k \leq(n-d-1) / 2$, the following question is open.

Problem 3.18. For which triples $d, k, n$ of integers with $2 \leq d \leq n-2$ and $3 \leq k \leq$ $(n-d-1) / 2$ does there exist a connected graph of order $n$ having diameter $d$ and acyclic partition dimension $k$ ? 
We have seen that if $G$ is a connected graph with $a(G)=a$ and $\operatorname{apd}(G)=b$, then $1 \leq a \leq b$ and $b \geq 2$. Next, we study those pairs $a, b$ of integers with $1 \leq a \leq b$ and $b \geq 2$ that are realizable as the vertex-arboricity and acyclic partition dimension of some connected graph. Since trees are the only connected graphs having the vertex-arboricity 1 , it follows that

$$
a(T) \neq \operatorname{apd}(T)
$$

for all trees $T$. Thus we may assume that $a \geq 2$. It is known that if $G$ is a connected graph of order $n$, then $a(G) \leq\lceil n / 2\rceil$. Thus, if $\operatorname{apd}(G)>\lceil n / 2\rceil$, then $a(G) \neq \operatorname{apd}(G)$. It is known that each pair $a, b$ of integers with $2 \leq a \leq b-1$ is realizable as the vertexarboricity and acyclic partition dimension of some connected graph.

THEOREM 3.19. For each pair $a, b$ of integers with $2 \leq a \leq b-1$, there exists $a$ connected graph $G$ with $a(G)=a$ and $\operatorname{apd}(G)=b$.

However, it is not known whether there exists a connected graph $G$ with $a(G)=$ $\operatorname{apd}(G)$.

If $H$ is an induced subgraph of a graph $G$, then $a(H) \leq a(G)$. Thus

$$
0<\frac{a(H)}{a(G)} \leq 1
$$

for each induced subgraph $H$ of a graph $G$. Let $G$ be a nontrivial connected graph and $H$ a connected induced subgraph of $G$. The acyclic partition ratio of $H$ and $G$ is defined by

$$
r_{a}(H, G)=\frac{\operatorname{apd}(H)}{\operatorname{apd}(G)} .
$$

Unlike the ratio in (3.21), it need not occur that $r_{a}(H, G) \leq 1$. Since apd $\left(K_{1, m}\right)=m$ for all $m \geq 2, G=K_{1, m}$, and $H=K_{2}$, we can make the ratio $r_{a}(H, G)$ as small as we wish by choosing $m$ arbitrarily large. Although this may not be surprising, it may be unexpected that, in fact, $r_{a}(H, G)$ can be as large as we wish (see [49]).

THEOREM 3.20. Let $\epsilon$ and $M$ be two real numbers.

(1) There exist a connected graph $G$ and an induced subgraph $H$ of $G$ such that $r_{a}(H, G)<\epsilon$.

(2) There exist a connected graph $G^{\prime}$ and an induced subgraph $H^{\prime}$ of $G^{\prime}$ such that $r_{a}\left(H^{\prime}, G^{\prime}\right)>M$.

4. Conditional resolving decompositions. A decomposition of a graph $G$ is a collection of subgraphs of $G$, none of which has isolated vertices, whose edge sets provide a partition of $E(G)$. A decomposition into $k$ subgraphs is a $k$-decomposition. A decomposition $\mathscr{D}=\left\{G_{1}, G_{2}, \ldots, G_{k}\right\}$ is ordered if the ordering $\left(G_{1}, G_{2}, \ldots, G_{k}\right)$ has been imposed on $\mathscr{D}$. If each subgraph $G_{i}(1 \leq i \leq k)$ is isomorphic to a graph $H$, then $\mathscr{D}$ is called an $H$-decomposition of $G$. Decompositions of graphs have been the subject of many studies. 
For edges $e$ and $f$ in a connected graph $G$, the distance $d(e, f)$ between $e$ and $f$ is the minimum nonnegative integer $k$ for which there exists a sequence $e=e_{0}, e_{1}, \ldots, e_{k}=f$ of edges of $G$ such that $e_{i}$ and $e_{i+1}$ are adjacent for $i=0,1, \ldots, k-1$. Thus, $d(e, f)=0$ if and only if $e=f, d(e, f)=1$ if and only if $e$ and $f$ are adjacent, and $d(e, f)=2$ if and only if $e$ and $f$ are nonadjacent edges that are adjacent to a common edge of $G$. Also, this distance equals the standard distance between vertices $e$ and $f$ in the line graph $L(G)$. For an edge $e$ of $G$ and a subgraph $F$ of $G$, we define the distance between $e$ and $F$ as

$$
d(e, F)=\min _{f \in E(F)} d(e, f) .
$$

Let $\mathscr{D}=\left\{G_{1}, G_{2}, \ldots, G_{k}\right\}$ be an ordered $k$-decomposition of a connected graph $G$. For $e \in E(G)$, the $\mathscr{D}$-code (or simply the code) of $e$ is the $k$-vector

$$
c_{\mathscr{D}}(e)=\left(d\left(e, G_{1}\right), d\left(e, G_{2}\right), \ldots, d\left(e, G_{k}\right)\right) .
$$

Hence exactly one coordinate of $c_{\mathscr{D}}(e)$ is 0 , namely, the $i$ th coordinate if $e \in E\left(G_{i}\right)$. The decomposition $\mathscr{D}$ is said to be a resolving decomposition for $G$ if every two distinct edges of $G$ have distinct $\mathscr{D}$-codes. The minimum $k$ for which $G$ has a resolving $k$-decomposition is its decomposition dimension $\operatorname{dim}_{d}(G)$. A resolving decomposition of $G$ with $\operatorname{dim}_{d}(G)$ elements is a minimum resolving decomposition for $G$.

To illustrate these concepts, consider the graph $G$ of Figure 4.1. Let $\mathscr{D}=\left\{G_{1}, G_{2}, G_{3}\right\}$, where $E\left(G_{1}\right)=\left\{e_{1}, e_{5}, f_{1}, f_{5}, f_{4}\right\}, E\left(G_{2}\right)=\left\{e_{2}, e_{3}, f_{2}\right\}$, and $E\left(G_{3}\right)=\left\{e_{4}, e_{6}, f_{3}, f_{6}, f_{7}\right\}$. The D-codes of the edges of $G$ are

$$
\begin{array}{llll}
\mathcal{C}_{\mathscr{D}}\left(e_{1}\right)=(0,1,2), & \mathcal{C}_{\mathscr{D}}\left(e_{2}\right)=(1,0,2), & \mathcal{C}_{\mathscr{D}}\left(e_{3}\right)=(2,0,1), & \mathcal{C}_{\mathscr{D}}\left(e_{4}\right)=(2,1,0), \\
\mathcal{C}_{\mathscr{D}}\left(e_{5}\right)=(0,4,1), & \mathcal{C}_{\mathscr{D}}\left(e_{6}\right)=(1,4,0), & \mathcal{C}_{\mathscr{D}}\left(f_{1}\right)=(0,1,1), & \mathcal{C}_{\mathscr{D}}\left(f_{2}\right)=(1,0,1), \\
\mathcal{C}_{\mathscr{D}}\left(f_{3}\right)=(1,1,0), & \mathcal{C}_{\mathscr{D}}\left(f_{4}\right)=(0,2,1), & \mathcal{C}_{\mathscr{D}}\left(f_{5}\right)=(0,3,1), & \mathcal{C}_{\mathscr{D}}\left(f_{6}\right)=(1,3,0), \\
\mathcal{C}_{\mathscr{D}}\left(f_{7}\right)=(1,2,0) . & &
\end{array}
$$

Thus, $\mathscr{D}$ is a resolving decomposition of $G$. In fact, there is no 2-element resolving

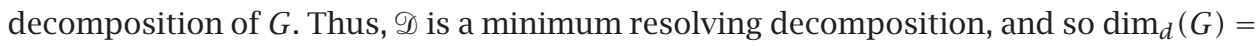
$|\mathscr{D}|=3$.

These concepts were first introduced in [7] and studied further in [25, 26, 35, 36].

4.1. Connected resolving decompositions. A resolving decomposition $\mathscr{D}=\left\{G_{1}\right.$, $\left.G_{2}, \ldots, G_{k}\right\}$ of a connected graph $G$ is connected if each subgraph $G_{i}(1 \leq i \leq k)$ is a connected subgraph in $G$. The minimum $k$ for which $G$ has a connected resolving $k$-decomposition is its connected decomposition dimension $\operatorname{cdim}_{d}(G)$. A connected resolving decomposition of $G$ with $\operatorname{cdim}_{d}(G)$ elements is called a minimum connected resolving decomposition of $G$. These concepts were introduced and studied in [40, 44]. If $G$ has $m \geq 2$ edges, then the $m$-decomposition $\mathscr{D}=\left\{G_{1}, G_{2}, \ldots, G_{m}\right\}$, where each set $E\left(G_{i}\right)(1 \leq i \leq m)$ consists of a single edge, is a connected resolving decomposition of $G$. Thus, $\operatorname{cdim}_{d}(G)$ is defined for every connected graph $G$ of size at least 2. Moreover, every connected resolving $k$-decomposition is a resolving $k$-decomposition, and 


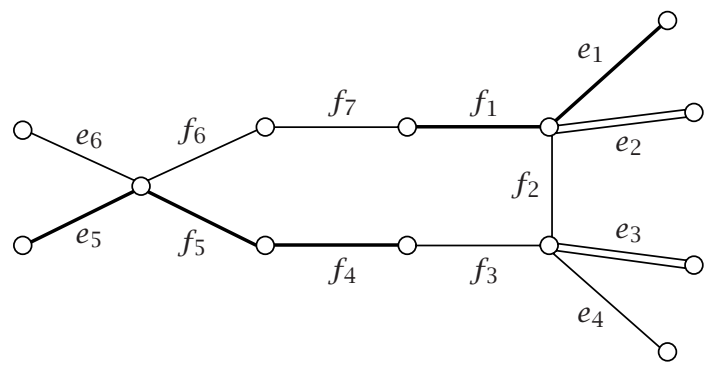

Figure 4.1. A graph $G$ with $\operatorname{dim}_{d}(G)=3$.

so

$$
2 \leq \operatorname{dim}_{d}(G) \leq \operatorname{cdim}_{d}(G) \leq m
$$

for every connected graph $G$ of size $m \geq 2$. Furthermore, all connected graphs $G$ of size $m \geq 4$ with $\operatorname{cdim}_{d}(G) \in\{2, m-2, m-1, m\}$ are characterized in [40].

The next three results [40] present bounds for $\operatorname{cdim}_{d}(G)$ of a connected graph $G$ in terms of (1) its size and diameter, (2) its size and girth, (3) its order.

THEOREM 4.1. If $G$ is a connected graph of size $m \geq 2$ and diameter $d$, then

$$
1+\log _{d+1} m \leq \operatorname{cdim}_{d}(G) \leq m-d+2 .
$$

The upper bound in Theorem 4.1 is sharp for $d \geq 2$, while the sharpness of the lower bound in Theorem 4.1 is unknown.

THEOREM 4.2. If $G$ is a connected graph of size $m \geq 3$ and girth $\ell \geq 3$, then

$$
3 \leq \operatorname{cdim}_{d}(G) \leq m-\ell+3 .
$$

Moreover, $\operatorname{cdim}_{d}(G)=m-\ell+3$ if and only if $G$ is a cycle of order at least 3 .

For a connected graph $G$, let

$$
m_{k}(G)=\min \{k(G-T): T \text { is a spanning tree of } G\},
$$

where $k(G-T)$ is the number of components of $G-T$.

THEOREM 4.3. If $G$ is a connected graph of order $n \geq 5$, then

$$
\operatorname{cdim}_{d}(G) \leq n+m_{k}(G)-1
$$

The upper bound in Theorem 4.3 is attainable for stars. On the other hand, strict inequality in Theorem 4.3 can hold as well.

The decomposition dimensions of trees that are not paths have been studied in $[7,26]$, where bounds for them have been determined. However, there is no general formula for the decomposition dimension of a tree that is not a path. On the other 
hand, a formula for the connected decomposition dimension of a tree that is not a path was established in [44].

THEOREM 4.4. If $T$ is a tree that is not a path, then

$$
\operatorname{cdim}_{d}(T)=\sigma(T)-\operatorname{ex}(T)+1
$$

Therefore, by Theorem 3.4, if $T$ is a tree that is not a path, then

$$
\operatorname{cpd}(T)=\operatorname{cdim}_{d}(T)=\sigma(T)-\operatorname{ex}(T)+1 .
$$

On the other hand, it was shown in [41] that

$$
\operatorname{cpd}(G) \geq \sigma(G)-\operatorname{ex}(G)+1
$$

for every connected graph $G$, while this is not true in general for connected decomposition dimensions of graphs (see [44]).

We have seen that if $G$ is a connected graph of size at least 2 with $\operatorname{dim}_{d}(G)=a$ and $\operatorname{cdim}_{d}(G)=b$, then $2 \leq a \leq b$. Furthermore, the paths of order at least 3 are the only connected graphs $G$ of size at least 2 with $\operatorname{dim}_{d}(G)=\operatorname{cdim}_{d}(G)=2$. Thus there is no connected graph $G$ with $\operatorname{dim}_{d}(G)=2$ and $\operatorname{cdim}_{d}(G)>2$. On the other hand, the following realization result appears in [44].

THEOREM 4.5. For every pair $a, b$ of integers with $3 \leq a \leq b$, there exists a connected graph $G$ such that $\operatorname{dim}_{d}(G)=a$ and $\operatorname{cdim}_{d}(G)=b$.

For a connected graph $G$ of size $m \geq 2$, the decomposition dimension ratio $r_{\mathrm{dim}}(G)$ of $G$ and the connected decomposition dimension ratio $r_{\mathrm{cd}}(G)$ of $G$ are defined as

$$
r_{\operatorname{dim}}(G)=\frac{\operatorname{dim}_{d}(G)}{m}, \quad r_{\mathrm{cd}}(G)=\frac{\operatorname{cdim}_{d}(G)}{m} .
$$

Since $2 \leq \operatorname{dim}_{d}(G) \leq \operatorname{cdim}_{d}(G) \leq m$ for every connected graph $G$ of size $m \geq 2$, it follows that

$$
0<r_{\text {dim }}(G) \leq r_{\text {cd }}(G) \leq 1
$$

Furthermore, $r_{\operatorname{dim}}(G)=1$ if and only if $r_{\mathrm{cd}}(G)=1$.

There exist infinitely many triples $a, b, m$ of integers with $2 \leq a \leq b \leq m$ that are not realizable as the decomposition dimension, connected decomposition dimension, and size of any connected graph. However, it was shown in [40] that every pair $s, t$ of rational numbers with $0<s \leq t<1$ is realizable as the decomposition dimension ratio and connected decomposition dimension ratio for some connected graph.

THEOREM 4.6. For each pair $s, t$ of rational numbers with $0<s \leq t<1$, there is a connected graph $G$ such that $r_{\mathrm{dim}}(G)=s$ and $r_{\mathrm{cd}}(G)=t$.

4.2. Independent resolving decompositions. A decomposition $\mathscr{D}=\left\{G_{1}, G_{2}, \ldots, G_{k}\right\}$ of a connected graph $G$ is called independent if $E\left(G_{i}\right)$ is independent for each $i$ ( $1 \leq$ $i \leq k)$ in $G$. This concept can be considered from an edge-coloring point of view. Recall 
that a proper edge coloring (or simply, an edge coloring) of a nonempty graph $G$ is an assignment $c$ of colors (positive integers) to the edges of $G$ so that adjacent edges are colored differently, that is, $c: E(G) \rightarrow \mathbb{N}$ is a mapping such that $c(e) \neq c(f)$ if $e$ and $f$ are adjacent edges of $G$. The minimum $k$ for which there is an edge coloring of $G$ using $k$ distinct colors is called the edge chromatic number $\chi_{e}(G)$ of $G$. If $\mathscr{D}=\left\{G_{1}, G_{2}, \ldots, G_{k}\right\}$ is an independent decomposition of a graph $G$, then by assigning color $i$ to all edges in $G_{i}$ $(1 \leq i \leq k)$, we obtain an edge coloring of $G$ using $k$ distinct colors. On the other hand, if $c$ is an edge coloring of a connected graph $G$, using the colors $1,2, \ldots, k$ for some positive integer $k$, then $c(e) \neq c(f)$ for adjacent edges $e$ and $f$ in $G$. Equivalently, $c$ produces a decomposition $\mathscr{D}$ of $E(G)$ into color classes (independent sets) $C_{1}, C_{2}, \ldots, C_{k}$, where the edges of $C_{i}$ are colored $i$ for $1 \leq i \leq k$. Thus, for an edge $e$ in a graph $G$, the $k$-vector

$$
c_{\mathscr{D}}(e)=\left(d\left(e, C_{1}\right), d\left(e, C_{2}\right), \ldots, d\left(e, C_{k}\right)\right)
$$

is called the color code (or simply the code) $c_{\mathscr{D}}(e)$ of $e$. If distinct edges of $G$ have distinct color codes, then $c$ is called a resolving edge coloring (or independent resolving decomposition) of $G$. Thus a resolving edge coloring of $G$ is an edge coloring that distinguishes all edges of $G$ in terms of their distances from the resulting color classes. A minimum resolving edge coloring uses a minimum number of colors, and this number is the resolving edge chromatic number $\chi_{\mathrm{re}}(G)$ of $G$. These concepts were introduced and studied in $[17,47]$.

Suppose that $E(G)=\left\{e_{1}, e_{2}, \ldots, e_{m}\right\}$, where $m \geq 2$. By assigning color $i$ to $e_{i}$ for $1 \leq i \leq m$, we obtain a resolving edge coloring of $G$. Thus, $\chi_{\mathrm{re}}(G)$ is defined for every graph $G$. Moreover, every resolving edge coloring is an edge coloring and every resolving edge coloring is a resolving decomposition. Therefore,

$$
2 \leq \max \left\{\operatorname{dim}_{d}(G), \chi_{e}(G)\right\} \leq \chi_{\mathrm{re}}(G) \leq m
$$

for each connected graph $G$ of size $m \geq 2$. It was shown in [17] that every pair $k, m$ of integers with $3 \leq k \leq m$ is realizable as the resolving edge chromatic number and size of some connected graph. Furthermore, characterizations of all connected graphs $G$ with $\chi_{\mathrm{re}}(G)=2,3, m$ have been established in [17].

THEOREM 4.7. Let $G$ be a connected graph of order $n \geq 3$ and size $m$. Then

(a) $\chi_{\mathrm{re}}(G)=2$ if and only if $G=P_{3}$;

(b) $\chi_{\mathrm{re}}(G)=3$ if and only if $n \geq 4$ and (i) $G=P_{n}$, (ii) $G=C_{n}$, where $n$ is odd, or (iii) $G \in \mathcal{T} \cup U$, where $\mathcal{T}$ is the set of all trees $T$ with $\Delta(T)=3$ having exactly one vertex of degree 3 and $U$ is the set of all unicyclic graphs $G$ with $\Delta(G)=3$ having an even cycle and exactly one vertex of degree 3;

(c) $\chi_{\mathrm{re}}(G)=m$ if and only if $G \in\left\{K_{3}, P_{4}, \overline{P_{3} \cup K_{1}}, C_{4}, K_{4}-e, K_{4}\right\}$ or $n \geq 5$ and $G=$ $K_{1, n-1}$.

Resolving edge chromatic numbers of paths, stars, and cycles were determined in [17] and the edge chromatic numbers of complete graphs and trees were studied in $[17,47]$, where upper bounds for the resolving edge chromatic number of a connected 
graph are established in terms of (1) its size and diameter, (2) its size and girth, (3) its order and edge chromatic number, (4) its decomposition dimension and edge chromatic number.

THEOREM 4.8. If $G$ is a connected graph of size $m \geq 3$ and diameter $d$, then

$$
\chi_{\mathrm{re}}(G) \leq m-d+3 \text {. }
$$

Moreover, $\chi_{\mathrm{re}}(G)=m-d+3$ if and only if $G$ is a path of size $m \geq 3$.

THEOREM 4.9. If $G$ is a connected graph of size $m$ and girth $\ell$, where $m \geq \ell \geq 3$, then

$$
\chi_{\mathrm{re}}(G) \leq \begin{cases}m-\ell+3 & \text { if } \ell \text { is odd } \\ m-\ell+4 & \text { if } \ell \text { is even }\end{cases}
$$

Furthermore, $\chi_{\mathrm{re}}(G)=m-\ell+4$ if and only if $G=C_{n}$ for some even $n \geq 4$.

If $G=C_{n}$ for some odd integer $n$, then $\ell=m=n$, and so $\chi_{\text {re }}(G)=m-\ell+3$. However, the odd cycles are not the only connected graphs $G$ of size $m \geq 3$ having girth $\ell \geq 3$ and $\chi_{\mathrm{re}}(G)=m-\ell+3$.

THEOREM 4.10. If $G$ is a connected graph of order $n \geq 5$, then

$$
\chi_{e}(G) \leq \chi_{\mathrm{re}}(G) \leq n+\chi_{e}(G)-1 .
$$

THEOREM 4.11. If $G$ is a connected graph of order $n \geq 5$, then

$$
\chi_{\mathrm{re}}(G) \leq n+k-1,
$$

where $k=\min \left\{\chi_{e}(\langle E(G)-E(T)\rangle): T\right.$ is a spanning tree of $\left.G\right\}$.

It is known that $\Delta(G) \leq \chi_{e}(G) \leq \Delta(G)+1$ for every nonempty graph $G$, where the upper bound is due to Vizing [53]. Thus, if $G$ is a connected graph of order $n \geq 5$, then

$$
\Delta(G) \leq \chi_{\mathrm{re}}(G) \leq n+\Delta(G) .
$$

However, just how good the upper bounds are in Theorems 4.10 and 4.11 and (4.20) is not known.

\section{REFERENCES}

[1] R. C. Brigham, G. Chartrand, R. D. Dutton, and P. Zhang, Isometric embeddings of bipartite graphs, Congr. Numer. 154 (2002), 7-12.

[2] _ Resolving domination in graphs, Math. Bohem. 128 (2003), no. 1, 25-36.

[3] P. Buczkowski, G. Chartrand, C. Poisson, and P. Zhang, On k-dimensional graphs and their bases, Period. Math. Hungar. 46 (2003), no. 1, 9-15.

[4] G. Chartrand, L. Eroh, M. A. Johnson, and O. R. Oellermann, Resolvability in graphs and the metric dimension of a graph, Discrete Appl. Math. 105 (2000), no. 1-3, 99-113.

[5] G. Chartrand, D. Erwin, M. A. Henning, P. J. Slater, and P. Zhang, The locating-chromatic number of a graph, Bull. Inst. Combin. Appl. 36 (2002), 89-101.

[6] __ Graphs of order $n$ with locating-chromatic number n-1, Discrete Math. 269 (2003), no. 1-3, 65-79. 
[7] G. Chartrand, D. Erwin, M. Raines, and P. Zhang, The decomposition dimension of graphs, Graphs Combin. 17 (2001), no. 4, 599-605.

[8] G. Chartrand, D. Erwin, P. J. Slater, and P. Zhang, Distance-location numbers of graphs, Util. Math. 63 (2003), 65-79.

[9] G. Chartrand, T. W. Haynes, M. A. Henning, and P. Zhang, Stratified claw domination in prisms, J. Combin. Math. Combin. Comput. 33 (2000), 81-96.

[10] - Stratification and domination in graphs, Discrete Math. 272 (2003), no. 2-3, 171185.

[11] G. Chartrand and H. V. Kronk, The point-arboricity of planar graphs, J. London Math. Soc. 44 (1969), 612-616.

[12] G. Chartrand, H. V. Kronk, and C. E. Wall, The point-arboricity of a graph, Israel J. Math. 6 (1968), 169-175.

[13] G. Chartrand and L. Lesniak, Graphs \& Digraphs, 3rd ed., Chapman \& Hall, London, 1996.

[14] G. Chartrand, C. Poisson, and P. Zhang, Resolvability and the upper dimension of graphs, Comput. Math. Appl. 39 (2000), no. 12, 19-28.

[15] G. Chartrand, M. Raines, and P. Zhang, The directed distance dimension of oriented graphs, Math. Bohem. 125 (2000), no. 2, 155-168.

[16] _ On the dimension of oriented graphs, Util. Math. 60 (2001), 139-151.

[17] G. Chartrand, V. Saenpholphat, and P. Zhang, Resolving edge colorings in graphs, to appear in Ars Combin.

[18] _ The independent resolving number of a graph, Math. Bohem. 128 (2003), no. 4, 379-393.

[19] G. Chartrand, E. Salehi, and P. Zhang, The partition dimension of a graph, Aequationes Math. 59 (2000), no. 1-2, 45-54.

[20] G. Chartrand and P. Zhang, On the chromatic dimension of a graph, Congr. Numer. 145 (2000), 97-108.

[21] _ The forcing dimension of a graph, Math. Bohem. 126 (2001), no. 4, 711-720.

[22] - The theory and applications of resolvability in graphs. A survey, Congr. Numer. 160 (2003), 47-68.

[23] G. Chartrand, P. Zhang, and E. Salehi, On the partition dimension of a graph, Congr. Numer. 130 (1998), 157-168.

[24] J. Currie and O. R. Oellermann, The metric dimension and metric independence of a graph, J. Combin. Math. Combin. Comput. 39 (2001), 157-167.

[25] H. Enomoto, Upper bound of the decomposition dimension of a graph, Congr. Numer. 145 (2000), 157-160.

[26] H. Enomoto and T. Nakamigawa, On the decomposition dimension of trees, Discrete Math. 252 (2002), no. 1-3, 219-225.

[27] M. R. Garey and D. S. Johnson, Computers and Intractability. A Guide to the Theory of NP-Completeness, A Series of Books in the Mathematical Sciences, W. H. Freeman, California, 1979.

[28] F. Harary and R. A. Melter, On the metric dimension of a graph, Ars Combin. 2 (1976), 191-195.

[29] B. L. Hulme, A. W. Shiver, and P. J. Slater, Fire: a Subroutine for Fire Protection Network Analysis, Sandia National Laboratories, New Mexico, 1981, SAND 81-1261.

[30] _ Computing Minimum Cost Fire Protection, Sandia National Laboratories, New Mexico, 1982, SAND 82-0809.

[31] _ A Boolean algebraic analysis of fire protection, Algebraic and Combinatorial Methods in Operations Research, North-Holland Math. Stud., vol. 95, North-Holland Publishing, Amsterdam, 1984, pp. 215-227.

[32] M. A. Johnson, Structure-activity maps for visualizing the graph variables arising in drug design, J. Biopharm. Statist 3 (1993), 203-236.

[33] _ Browsable structure-activity datasets, Advances in Molecular Similarity (R. CarbóDorca and P. Mezey, eds.), JAI Press, Connecticut, 1998, pp. 153-170. 
[34] S. Khuller, B. Rsghavachari, and A. Rosenfeld, Localization in graphs, Tech. report, University of Maryland, Maryland, 1994.

[35] A. Küngen and D. B. West, Decomposition dimension of graphs and a union-free family of sets, preprint, 2001.

[36] T. Nakamigawa, A note on the decomposition dimension of complete graphs, Ars Combin. 69 (2003), 161-163.

[37] C. Poisson and P. Zhang, The metric dimension of unicyclic graphs, J. Combin. Math. Combin. Comput. 40 (2002), 17-32.

[38] M. Raines and P. Zhang, The Steiner distance dimension of graphs, Australas. J. Combin. 20 (1999), 133-143.

[39] D. F. Rall and P. J. Slater, On location-domination numbers for certain classes of graphs, Congr. Numer. 45 (1984), 97-106.

[40] V. Saenpholphat and P. Zhang, On connected resolving decompositions in graphs, to appear in Czechoslovak Math. J.

[41] C Connected partition dimensions of graphs, Discuss. Math. Graph Theory 22 (2002), no. 2, 305-323.

[42] _ Some results on connected resolvability in graphs, Congr. Numer. 158 (2002), 5-19.

[43] _ Connected resolvability of graphs, Czechoslovak Math. J. 53(128) (2003), no. 4, 827-840.

[44] _ Connected resolving decompositions in graphs, Math. Bohem. 128 (2003), no. 2, 121-136.

[45] _ _ Connected resolving sets in graphs, Ars Combin. 68 (2003), 3-16.

[46] _ On connected resolvability of graphs, Australas. J. Combin. 28 (2003), 25-37.

[47] _ _ On resolving edge colorings in graphs, Int. J. Math. Math. Sci. (2003), no. 46, 29472959.

[48] _ On resolving acyclic partitions of graphs, Ars Combin. 71 (2004), 65-78.

[49] _ Resolving acyclic partitions of graphs, Ars Combin. 70 (2004), 61-74.

[50] P. J. Slater, Leaves of trees, Proc. 6th Southeastern Conference on Combinatorics, Graph Theory, and Computing (Florida Atlantic Univ., Boca Raton, Fla, 1975), Congressus Numerantium, no. 14, Utilitas Math., Winnipeg, Manitoba, 1975, pp. 549-559.

[51] _ Domination and location in acyclic graphs, Networks 17 (1987), no. 1, 55-64.

[52] _ _ Dominating and reference sets in a graph, J. Math. Phys. Sci. 22 (1988), no. 4, 445455.

[53] V. G. Vizing, On an estimate of the chromatic class of a p-graph, Diskret. Analiz No. 3 (1964), 25-30.

Varaporn Saenpholphat: Department of Mathematics, Srinakharinwirot University, Bangkok, 10110, Thailand

E-mail address: varaporn@swu.ac.th

Ping Zhang: Department of Mathematics, Western Michigan University, Kalamazoo, MI 49008, USA

E-mail address: ping . zhang@wmich .edu 


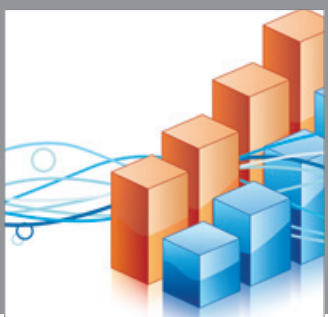

Advances in

Operations Research

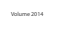

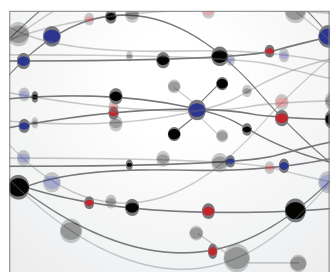

\section{The Scientific} World Journal
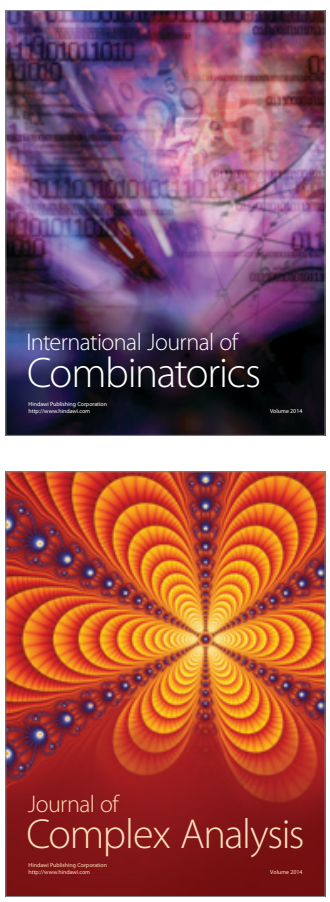

International Journal of

Mathematics and

Mathematical

Sciences
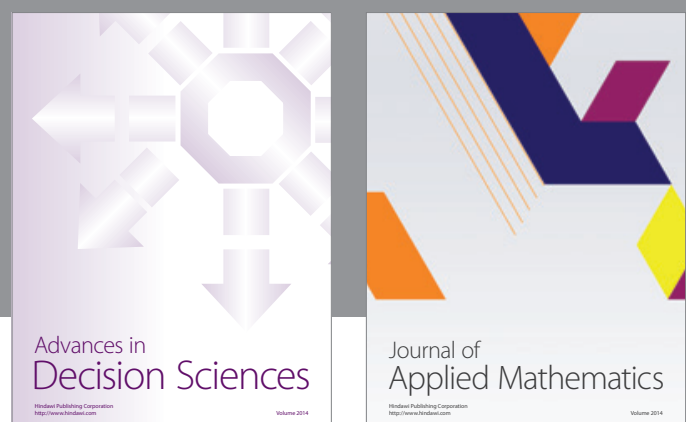

Journal of

Applied Mathematics
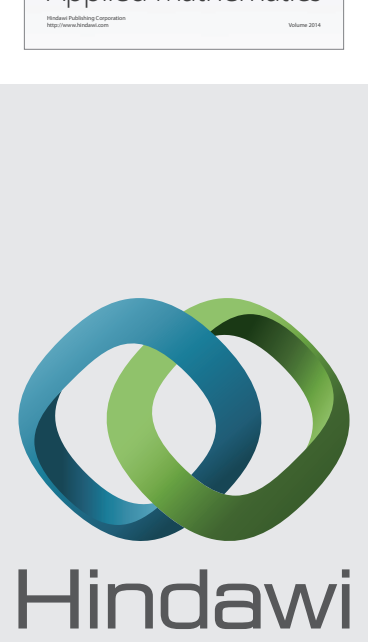

Submit your manuscripts at http://www.hindawi.com
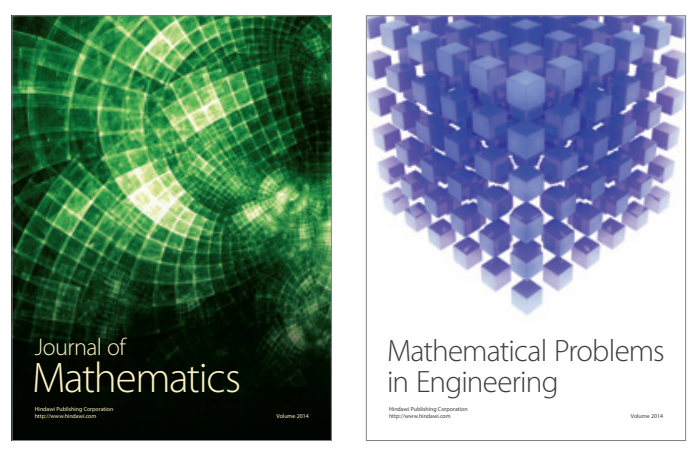

Mathematical Problems in Engineering
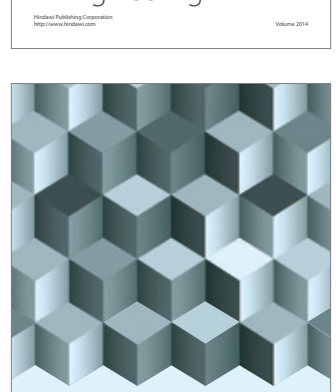

Journal of

Function Spaces
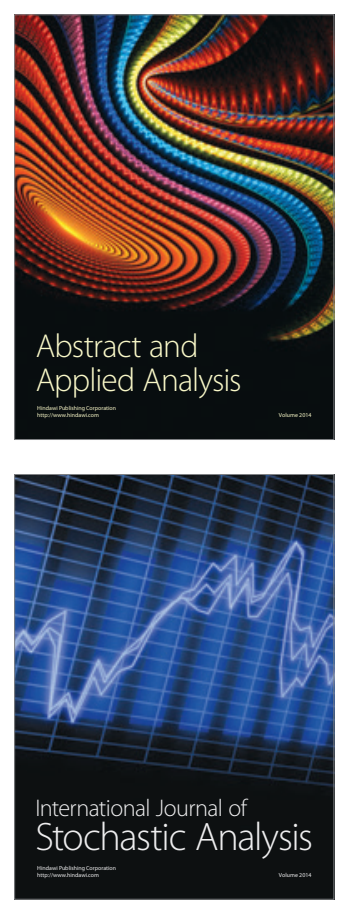

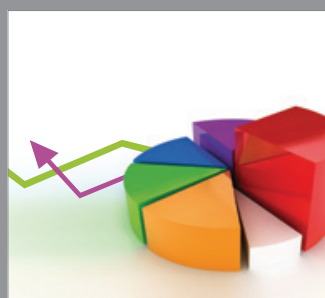

ournal of

Probability and Statistics

Promensencen
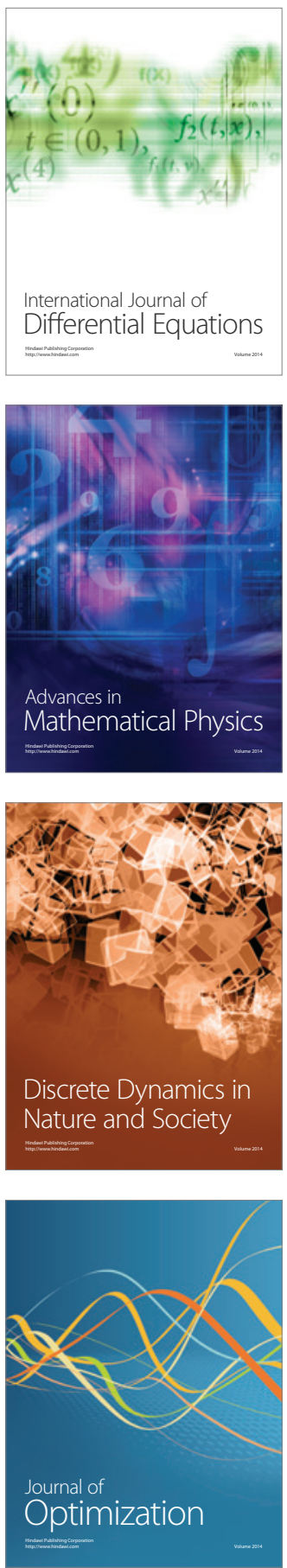